\title{
Exploring the Use of Immersive Virtual Reality to enhance psychological wellbeing in Pediatric
}

\section{Oncology}

\section{Authors}

Michelle, Tennant ${ }^{1,2,3}$, George J. Youssef ${ }^{1,4}$, Jane A. McGillivray ${ }^{1,5}$, Tara-Jane Clark ${ }^{3}$, Laura McMillan $^{2}$, Maria C. McCarthy ${ }^{2,3,6}$

\section{Affiliations}

${ }^{1}$ School of Psychology, Deakin University, Geelong, Australia

${ }^{2}$ Clinical Sciences, Murdoch Children's Research Institute, Parkville, Australia

${ }^{3}$ Children's Cancer Centre, The Royal Children's Hospital, Parkville, Australia

${ }^{4}$ Centre for Adolescent Health, Murdoch Children's Research Institute, Melbourne, Australia

${ }^{5}$ Deakin Child Study Centre, School of Psychology, Deakin University, Burwood, Australia

${ }^{6}$ Department of Paediatrics, University of Melbourne, Parkville, 3052, Australia

Corresponding Author: Michelle Tennant ${ }^{1}$

${ }^{1}$ Michelle Tennant, Deakin University, School of Psychology, Faculty of Health, 221 Burwood Hwy, Burwood, VIC, 3125, Australia. Phone: +61408 127 717.E-mail: mtennan@ deakin.edu.au. 


\section{Author Statements}

Acknowledgements: We acknowledge the important contribution of the healthcare professionals, children and parents of the Royal Children's Hospital, Children's Cancer Centre who participated in this study, as well as the dedication and in kind support provided by immersive media company, Phoria.

Ethical standards: The authors assert that all procedures contributing to this work comply with the ethical standards of the relevant national and institutional committees on human experimentation and with the Helsinki Declaration of 1975, as revised in 2008.

Conflicts of interest: None. 


\begin{abstract}
Background Objectives: To investigate whether Immersive Virtual Reality (VR) has a greater positive influence on oncology patients' physical and emotional mood states when compared to an iPad attentional control condition. Our secondary objective was to understand what factors influenced VR effectiveness.

Method: Participants were 90 oncology inpatients, aged 7-19 years, and their primary parent caregiver. Using a randomised controlled study design patients were allocated to VR (three content groups) or an iPad control condition. Pre-post-intervention self-report state measures were collected using visual analogue scales and an objective measure of physiological arousal (pulse rate). Post-intervention, patients reported on level of immersion, enjoyment and simulator sickness.
\end{abstract}

Results: Patients benefited from both Immersive VR and novel iPad intervention with no statistically significant differences found between conditions on child outcomes. However, patients accessing Immersive VR consistently reported greater positive shifts in mood state and reductions in negative symptoms when compared with iPad. No change was observed in physiological arousal levels (pulse rate) in either condition before, during or immediately after intervention. Moderation analysis showed that the degree of child illness (PedsQL), sex, age, and level of immersion were important in influencing the magnitude of differences between the VR and iPad conditions on mood, anxiety and pain.

Conclusion: These preliminary findings support the use of Immersive VR in clinical oncology settings to improve patient wellbeing. Further studies examining the application of Immersive VR in supporting children adjusting to hospitalization and cancer treatment are therefore warranted. Factors found to moderate VR effectiveness provide important clinical implications.

Keywords: Adolescents, Children, Hospitalization, Intervention, Oncology, Psychological, Virtual Reality 


\section{Introduction}

Childhood cancer treatment is complex and involves many challenges, including multiple hospitalizations, invasive procedures, and adverse physical and psychological treatment side effects. Although many children and adolescents demonstrate psychological resilience (Van Schoors et al., 2015), research has shown treatment burden contributes to high levels of global psychological distress among pediatric patients and their families (Linder and Hooke, 2019, Hooke and Linder, 2019), which can lead to significant psychological symptoms into survivorship, including depression, anxiety, somatic symptoms, and post-traumatic stress symptoms (PTSS) (Rourke et al., 2015). There is a need, therefore, for targeted, effective interventions that support children in regaining or maintaining their emotional wellbeing and quality of life in the context of serious illness, and that improve the psychological late effects of pediatric cancer.

Immersive Virtual Reality (VR) is an emerging therapeutic tool that offers promise as an engaging intervention for children and adolescents in tertiary healthcare settings (Won et al., 2017). Typical VR systems use a head-mounted display (HMD) for an individual user, or computer automatic virtual environment (CAVE) for multiusers, to immerse participants' senses in realistic and compelling virtual environments. When full immersion in the virtual world is achieved, sensory information from the real world may not be perceived, resulting in a subjective feeling of 'presence' in the virtual environment (Slater and Wilbur, 1997). Beyond its entertainment or distraction value, research has shown VR to be a viable alternative to traditional psychological therapies, such as cognitive behavioral therapy and in vivo exposure in the treatment of anxiety disorders, eating disorders, phobias and PTSS (Valmaggia et al., 2016, Krijn et al., 2004); and in the treatment of psychological stress and pain (Kenney and Milling, 2016) .

In pediatric settings, the most widely researched clinical application of VR has focused on the effectiveness of VR distraction therapy (VR-DT) in the attenuation of acute pain, anxiety and distress during invasive medical procedures. These studies have demonstrated VR-DT to be superior over 
standard hospital distraction practices (e.g. toys, television, books, parental comforting) (Won et al., 2017, Chirico et al., 2016, Dascal et al., 2017). VR rehabilitation therapy (VR-RT) (i.e. interactive VR games) is another emerging field in pediatrics focused on patients with intense physical therapy needs, such as cerebral palsy or severe burns injury, with initial studies demonstrating effectiveness of VR-RT on several psychological targets, including enhanced motivation and engagement, mood and enjoyment in activities that may be otherwise painful, difficult or boring (Parsons et al., 2009).

A compelling and relatively underexplored application of VR in pediatric inpatient settings is the capacity for VR to be used as a substitute for activity and play that may be otherwise inaccessible to patients during hospitalization. Immersion in VR games/activity has been found to enhance feelings of self-efficacy and accomplishment in children with cerebral palsy, resulting in patients developing more positive self-image and enhanced mood during treatment (Parsons et al., 2009). In the first controlled study using VR CAVE in hospitalized children with cancer, Li et al (Li et al., 2011) found that patients receiving 30 minutes of daily immersion in VR therapeutic play reported statistically fewer depressive symptoms than children in the control group on day seven of an inpatient admission ( $\mathrm{Li}$ et al., 2011). These findings illustrate how the subjective experience of 'presence' in virtual environments may be used as a therapeutic substitute for natural play and exploration among children with chronic illness and may ameliorate the potential negative psychological reactions associated with hospitalization, while bolstering mood and quality of life.

The purpose of this pilot study was to examine the potential clinical utility of Immersive VR experiences in an inpatient pediatric oncology setting as a brief psychological intervention for enhancing patient wellbeing. The primary objective was to investigate, via a randomized controlled design, whether Immersive VR intervention had greater positive influence on children's physical (pulse rate, nausea, pain) and emotional states (anxiety, anger, mood) during an inpatient oncology admission, when compared to an iPad control condition. A secondary objective was to evaluate the influence of demographic (age, sex), treatment (length of admission, child functional status), 
psychological (trait-anxiety), and intervention specific variables (immersion level, VR content, enjoyment, simulator sickness) on child outcomes.

\section{Method}

\subsection{Approvals}

Ethics approval was granted by the Human Research Ethics Committee of the Royal Children's Hospital (RCH), Melbourne (HREC 36345). Informed written consents were obtained from the parents/caregivers, with verbal assent obtained from all participants.

\subsection{Design}

A single-blind (participant), randomized controlled experimental design was employed in accordance with CONSORT guidelines (Schulz et al., 2010), to compare the effectiveness of Immersive VR intervention with iPad control. Assignment followed a 2:1 ratio to treatment group (Immersive VR: Non-immersive iPad control) and a 1:1:1 ratio for content type (Animals: Nature: Travel).

\subsection{Participants and Setting}

Participants were recruited from the Children's Cancer Centre (CCC) at the Royal Children's Hospital (RCH), Melbourne, Australia, over an 18-month period between 2017 and 2019. Eligibility criteria included (i) oncology inpatients aged $7-19$ years on active cancer treatment, (ii) at least one month ( 28 days) post a new diagnosis, and (iii) assessed as medically stable by oncology nursing staff. Exclusion criteria included (a) parent not present to obtain consent; (b) parent or child's English insufficient to complete study questionnaires; (c) child with a significant neurological condition, or major developmental disability; (d) child receiving palliative care; or (e) child with active infection. The age range of $7-19$ years was applied because of the language comprehension skills required to complete study measures. Ninety patients with a mean age of 11.59 years $(S D=$ 3.34) were recruited. See Table 1 for sample characteristics. 


\subsection{Interventions}

\subsubsection{Immersive Virtual Reality}

Immersive VR experiences were provided using a smartphone (Galaxy S7®; Samsung) and VR headset (Samsung Gear VR® first-generation mobile HMD; released November 2015). Whilst wearing the VR HMD and over ear headphones, children were immersed in a 3D, 270-degree virtual environment (visual field and corresponding soundscape), removing them from the visual and auditory stimuli of their hospital room. Total headset weight (HMD + S7 device) was approximately 462-grams. Smartphone display resolution was 1440 x 2560 pixels, 16:9 ratio ( $\sim 577$ ppi density).

\subsubsection{Non-immersive iPad control}

Non-immersive experiences were provided using an iPad (Model A1475) and over ear headphones, to deliver identical content presented in the VR condition (i.e. interactive $3 \mathrm{D}, 360^{\circ}$ video format). Whilst using the equipment, children could still process incoming visual stimuli from their hospital room during content viewing.

\subsubsection{Intervention Content}

Original $360^{\circ}$ video content was produced in collaboration with a VR production company (Phoria, Melbourne, Australia). Participants viewed one of three 10-minute virtual simulation experiences, including simulated travel to Australian national parks (i.e. 'Nature' experience), Australian zoos (i.e. 'Animal' experience), or global city tourist spots (i.e. 'Travel' experience). The three themes were selected to suit the interests of a wide age-range of participants with sample content validated via user-testing with oncology healthcare professionals during the early stages of intervention development.

\subsection{Measures}

Measures and data collection time-points are summarized in Table 2. We measured the effects of intervention on common cancer-related emotional and physical distress symptoms. The primary outcomes of child emotional and physical states (mood, anxiety, anger, nausea, and pain) were 
obtained using child-report Visual Analogue Scales (VASs). An objective measure of physiological arousal (child pulse rate) was also obtained. Other measures assessed the hypothesized moderating factors of intervention effectiveness pertaining to the secondary outcomes (e.g. trait-anxiety, illness status [PedsQL], immersion, enjoyment, simulator sickness). Demographics and illness details were collected from parents and medical records. Completion of pre-and-post online measures and VASs were performed using an iPad, with data imported directly to a REDCap (Harris et al., 2009) database. Parent proxy-report was used to assess child illness status (PedsQL).

\subsection{Participant Blinding}

Children were informed of the researchers' interest in "seeing how children on this ward feel about different types of technology". They were then informed that participation would involve "watching an interactive experience using either a VR headset or on an iPad", viewing experiences that involved "seeing animals, being in nature or touring cities".

\subsection{Randomization}

Block randomization was by a computer-generated random number list prepared and uploaded to REDCap (Harris et al., 2009) by an investigator with no clinical involvement in the trial. Eligible participants were randomised to one of six potential groups (i.e., $3 x \mathrm{VR}+3 \mathrm{xiPad}$ ), using random block sizes of 3,6 , and 9 , stratified by age ( $<12$ years vs $>=12$ years) to ensure similar proportions of children and adolescents across the treatment conditions. Randomization was performed electronically at the scheduled time of participation following consent.

\subsection{Procedures}

The intervention was delivered by trained research assistants. Children completed pre-online measures and were fitted with a pulse oximeter with baseline pulse rate obtained before introducing the technology. Children in the VR condition received assistance to comfortably fit the HMD and headphones, then viewed a 2-minute instructional video that guided them through navigation within the virtual environment. Children in the iPad control condition were given a demonstration on how to 
navigate the $360^{\circ}$ video presented on screen. Pulse rate was recorded at the mid- and end-point of intervention use. Children completed post-online measures immediately following the intervention.

\subsection{Statistical Methods}

A regression-based analysis of covariance (ANCOVA) approach was used to examine the primary outcomes of interest. Specifically, we regressed the outcome (i.e., pulse rate, VASs), on intervention group (i.e., VR vs iPad experience), content type (i.e., nature vs animals vs travel), and baseline assessment of the outcome. Separate regression models were specified for each outcome, with each model adjusting for child age, sex, trait-anxiety, length of admission, and illness status. Secondary analyses investigated whether treatment group differences at post-intervention were moderated by immersion, enjoyment, age, sex, trait-anxiety, illness status, length of admission and content type. Stata 13 (StataCorp, 2013) was used for all statistical analyses. Given the exploratory nature of the study, we used an alpha threshold for statistical significance of 0.1 to reduce the possibility of type 2 error and identify potential effects worth investigating in future studies.

\section{Results}

\subsection{Baseline Characteristics of Intervention Groups}

The study CONSORT diagram is presented in supplementary material (see Figure S1). Overall, of the 93 consented participants 61 completed the VR intervention and 29 completed iPad control treatment. Three participants did not receive VR intervention as allocated, contrary to intention to treat and were subsequently excluded in the analysis (see supplementary, Figure S1 for reasons). Baseline sample characteristics appeared to be comparable between groups, including child age, trait anxiety, length of admission and illness status. A slightly higher proportion of males was observed in the VR group. 


\subsection{Efficacy of Immersive VR Therapy on Child Outcomes}

\subsubsection{VAS and Pulse rate following intervention}

Table 4 presents the mean scores of the primary outcome measures (i.e., VASs and pulse rate) at post-intervention for the VR and iPad groups after adjusting for baseline levels of each outcome, respectively, and content type, child age, sex, trait-anxiety, illness status and length of admission. Overall, when compared to the baseline assessment of these measures, all children demonstrated improvement in positive mood state and reductions in negative states of anxiety, anger, nausea and pain following VR and iPad intervention. Nevertheless, children who received Immersive VR consistently reported greater change across all VASs measures post-treatment when compared to iPad control. The effect sizes for these differences ranged from weak to moderate in strength but always favored the VR condition (see Table 4). The strongest effect size was found for the VR condition which resulted in a 0.38 standard deviation higher VAS positive mood score following treatment when compared to children in the iPad control condition. No change in physiological arousal levels (pulse rate) was observed in either condition (Table 4).

\subsubsection{Immersion and enjoyment with intervention}

Consistent with trends found in VASs data, children in the VR condition reported slightly greater immersion and enjoyment compared to iPad, however these differences were not statistically significant (see Table 4). Children indicated high levels of overall enjoyment, with mean scores $>8$ on a scale $0-10$ (no enjoyment—most enjoyment) in both conditions.

\subsection{Moderation of Intervention Outcomes}

Addressing the secondary objectives of this study, moderation analyses were conducted to explore the influence of child age, sex, length of admission, illness status, trait-anxiety, immersion, enjoyment, simulator sickness and content type on each of the primary outcomes effects after intervention (i.e., VASs, pulse rate). Figure 1 presents the findings of simple slopes analyses for the moderation effects found in graphical form. 
Among the intervention-specific factors evaluated, we found evidence that post-intervention group differences in VAS Anger was moderated by Immersion level (Fig 2a). In the VR condition, children who reported higher immersion showed larger decreases in anger $(b=-0.67, \mathrm{se}=0.21$, $p=0.002$ ), whereas there was no observed effect of immersion level on anger outcomes for children in the iPad condition $(b=-0.03$, $\mathrm{se}=0.26, \mathrm{p}=0.922)$.

In separate models, we found that post-intervention group differences in VAS Positive Mood was moderated by Age (Fig 2b), Illness Status (Fig 2c), and Sex (Fig 2d). In the VR condition, positive mood improvement was shown to be independent of child age $(b=-0.59, \mathrm{se}=0.62, \mathrm{p}=0.343)$. This was not replicated for the iPad condition, which showed decreased benefit to mood with age $(b=$ $-3.57, \mathrm{se}=1.21, \mathrm{p}=0.004)$. Children in the VR condition showed an expected benefit to positive mood with improved illness status (i.e. greater functional capacity based on PedsQL scores) $(b=-0.29$, $\mathrm{se}=0.16, \mathrm{p}=0.73$ ) but this was not observed in the iPad condition, which conversely showed reduced benefit with improving illness status and functional capacity $(b=0.37, s e=0.26, p=0.156)$. In terms of the effect of sex differences, there was no statistical difference for males on positive mood outcomes between $\mathrm{iPad}(\mathrm{m}=84.58, \mathrm{se}=4.94)$ and VR conditions $(\mathrm{m}=79.20, \mathrm{se}=4.93, \mathrm{p}=0.352)$. However, positive mood was higher in the VR condition $(\mathrm{m}=86.50, \mathrm{se}=3.51)$ than the iPad condition $(\mathrm{m}=68.56$, $\mathrm{se}=4.39, \mathrm{p}=0.002$ ) for females.

Sex differences also appeared to moderate post-intervention group differences in VAS Anxiety (Fig 2e) and VAS Pain (Fig 2f). There were no statistical differences for males on state anxiety outcomes between iPad $(\mathrm{m}=8.49, \mathrm{se}=4.24)$ and VR conditions $(\mathrm{m}=13.31, \mathrm{se}=2.43, \mathrm{p}=0.329)$. However, state anxiety was lower in VR $(\mathrm{m}=5.51$, se=3.02) compared to the iPad condition $(\mathrm{m}=13.99, \mathrm{se}=3.75, \mathrm{p}=0.083)$ for females. Similar patterns were observed in children's pain scores, with no difference in outcomes between iPad $(\mathrm{m}=10.45$, $\mathrm{se}=3.30)$ and VR conditions $(\mathrm{m}=12.44$, $\mathrm{se}=1.89, \mathrm{p}=.606)$ for males. Whereas pain scores were lower in $\mathrm{VR}$ condition $(\mathrm{m}=8.19, \mathrm{se}=2.36)$ compared to the iPad condition $(\mathrm{m}=15.52, \mathrm{se}=2.92, \mathrm{p}=0.056)$ for females. 
No moderation effects were found for enjoyment, trait anxiety and length of admission on any of the primary outcomes.

\subsubsection{Moderation of intervention outcomes by content type}

There was also little evidence to support any moderation effects of intervention content type on child outcomes; however graphical representation of the simple slopes analyses conducted presented some noteworthy trends in VAS Positive Mood (Fig 2g) and VAS Pain (Fig 2h) between treatment conditions. Upon visual inspection of Fig $2 \mathrm{~g}$, we see high levels of positive mood reported by children in the VR condition irrespective of content type, but this was not observed in the iPad condition which showed variation in mood outcomes between content conditions. In Fig $2 \mathrm{~h}$, we can see more variability in pain outcomes reported by children in the VR conditions across content conditions when compared to iPad, with best outcomes or least pain observed with VR Nature compared to other treatment conditions (i.e. VR Animals, VR Travel, iPad Nature, iPad Animals, iPad Travel).

\section{Discussion}

This pilot study explored the clinical utility of using Immersive VR experiences in the general inpatient oncology setting to support children's adjustment to hospitalization and coping with cancer treatment. Employing a randomized controlled design, we compared Immersive VR with an iPad control condition that presented identical content in a non-immersive format. The study also explored the influence of various patient factors and treatment factors, and intervention factors (i.e. content type, immersion level, VR-induced sickness) on VR effectiveness.

\subsection{Effectiveness of Immersive VR Intervention}

A clinically significant improvement in child wellbeing was shown across all subjective measures, as indicated by increased positive mood and reductions in negative symptoms states (i.e. anxiety, anger, pain, nausea) for both VR and iPad intervention, with no statistically significant 
differences $(p>.05)$ between the two treatment groups on any of the primary outcomes. The direction of the VR effects in evoking positive affect and alleviating cancer-related distress symptoms were consistent with those reported in previous pilot studies in pediatric hospital settings (Schneider and Workman, 1999, Sander Wint et al., 2002, Gershon et al., 2004, Wolitzky et al., 2005, Li et al., 2011).

On examination of the magnitude of change between pre-and-post intervention measurements, VR consistently outperformed iPad across all subjective measures that was suggestive of an effect of the VR intervention. The lack of statistically significant group differences may therefore be due to the smaller sample size in this pilot study. The lack of findings may also be explained using theories of attention that relate to the VR concept of immersion in examining the unexpected benefits demonstrated in the iPad control condition (Gold et al., 2007). By design, VR systems have greater immersive capacity, defined as sensory engagement, than any other medium, and can evoke a believable sense of 'presence' in the virtual world (Slater and Wilbur, 1997). Importantly, greater immersion has been associated with better outcomes (e.g. less pain) with attentional demands reducing perception of internal or external stressors outside of the virtual environment (Eijlers et al., 2019). Unexpectedly patients reported similar levels of subjective immersion between conditions, indicating strong psychological engagement or mental absorption with both. Thus, while in the iPad condition participants could more easily disengage by looking away from the virtual environment and attending to environmental stressors, high levels of reported immersion may explain the strength of the effects even in the iPad condition.

Of note, studies that have compared VR intervention to simpler, more traditional forms of hospital intervention (e.g. toys, music, books, parental/nurse comforting, no intervention) have found stronger effects for VR intervention (Eijlers et al., 2019). Future research efforts should therefore include multiple comparison groups that are representational of usual care as well as alternative technological interventions to determine the true effectiveness of VR in clinical settings. 
In the current study, the most significant indicator of VR effectiveness was a moderate sized alleviation of symptoms of low mood $(d=0.38, p=0.1)$. This finding is congruent with that reported by Li and colleagues, who demonstrated an effect of daily VR immersion (using multi-user VR CAVE) on the reduction of depressive symptoms over the course of a 7-day hospital oncology admission $\left(\eta p^{2}=0.06, p=0.02\right)$ (Li et al., 2011). Together, these preliminary observations suggest Immersive VR therapy may be an advantageous technique in supporting mood adjustment during long inpatient stays.

On other child states, including self-ratings of anxiety, anger, pain and nausea, the effect of the VR intervention were smaller ( $d=$ between 0.09 and 0.17 ), with no effect on children's physiological arousal within and between conditions. This may be due to the lack of severity of symptoms at baseline, as indicated in previous studies using VR-DT (Sander Wint et al., 2002, Gershon et al., 2004, Chan et al., 2007, Kipping et al., 2012, Jeffs et al., 2014, Gold et al., 2006). The minimal change in arousal levels evident during the VR and iPad conditions is congruent with the intervention occurring while patients were resting in bed, as opposed to during a feared needleprocedure or painful wound care where VR-DT has resulted in symptom reduction (Gershon et al., 2004, Wolitzky et al., 2005, Hua et al., 2015).

\subsection{Moderating influences on VR effectiveness}

To our knowledge, this study is unique in evaluating influences of Immersive VR efficacy within a pediatric oncology context. We found that immersion level moderated the relationship between group and anger outcomes, with a negative relationship between immersion and state anger only for the VR group. This finding lends support to the theories of immersion and attention explained earlier, in that full immersion in VR places a greater attentional load on the user, thereby leaving fewer cognitive resources to focus on anger (Slater and Wilbur, 1997). It is noteworthy that this relationship was not observed in the iPad condition, who had a generally low level of anger regardless of the level of immersion. This lends some support to a potential anger inducing effect for 
the VR group when they are not feeling immersed. It is possible that when children did not achieve high immersion in VR they became frustrated due to a lack of alternative stimuli to focus on, whereas in iPad children could simply detach from the experience by turning away from the screen and refocusing on something else. Consequently, this suggests an importance of alignment between VR content and/or comfort with using VR equipment and the user to increase their level of immersion.

Compared with iPad-based interactive virtual environments, overall the VR intervention resulted in larger increases in positive mood and alleviation of low mood symptoms that did not appear to be affected by patient age or sex. This is consistent with previous investigations of VR-DT in acute pain settings where a VR analgesic effect has been demonstrated to be independent of child age, sex and ethnicity (Sharar et al., 2007). This provides increasing evidence that Immersive VR is of widespread appeal and potential benefit in treating psychological symptoms within pediatric settings. However, there was evidence to suggest it is important to consider patients' illness health status, since more optimal results were found in those with higher functional status. This finding has implications for the clinical application of Immersive VR, suggesting that when children are most unwell VR maybe overwhelming for them. This is potentially due to the highly stimulating nature of VR, and the associated attentional and physical energy requirements needed to derive optimal benefits to mood.

Our findings also demonstrated a preference for VR by specific patient groups. Our results showed VR was a superior intervention for enhancing mood in adolescent patients (i.e. > 13 years) and female patients. It is possible that VR is a more compelling intervention for these sub-groups due to its highly immersive and interactive properties. By contrast, younger and male patients in this study showed mood improvement from both VR and iPad intervention, suggesting a broader effect of technology in general as a technique for relieving stress emotions and supporting mood adjustment in hospital. Congruent with previous research that has demonstrated reduced effectiveness of simpler 
hospital distraction techniques with older children or adolescents on psychological outcomes (Carlson et al., 2000), our results suggest VR is an effective, age-appropriate intervention for adolescents that has potential to enhance success with engaging them in psychological intervention in the treatment of low mood symptoms. Similar to mood outcomes, sex differences emerged with VR shown to be a superior intervention for reducing state anxiety and pain in females, while male patients were shown to benefit from both VR and iPad intervention. Further study is needed to validate these preliminary findings.

Finally, this study found little evidence to suggest an influence of content type (i.e., nature, animals, travel) on child outcomes. This allows us to speculate that the unique experience of being believably immersed in novel virtual environments and the opportunity to engage children in leisure activity, exploration and play that is created within these environments, is effective in temporarily reducing emotional stress symptoms and enhancing mood during hospitalisation. Whereas, less immersive technologies or forms of distraction (e.g. tv, stories) may rely more on matching of patients' idiosyncratic interests with the focal stimuli to achieve similar levels of mental absorption required to achieve desired effects. However, regarding children's pain outcomes there was a slight suggestion of a greater effect of VR nature content on pain outcomes, aligning with previous investigations that have demonstrated positive effects of virtual contact with nature on psychological variables (McAllister et al., 2017). Overall, continued investigation is needed to further explain the role of VR content on proving optimal outcomes when targeting different psychological and physical symptoms.

\subsection{Clinical implications}

Despite high rates of clinically significant distress in pediatric cancer, there is a relative deficit of developmentally appropriate and effective interventions to help children and adolescents to cope with the stress and isolation of hospitalization and significant side-effects of treatment. The results of this study suggest Immersive VR may be a valuable resource as a brief intervention to 
ameliorate patients' negative psychological states. The high prevalence of persistent 'sadness' identified across other studies during the early phases of pediatric cancer treatment (Linder and Hooke, 2019) provide a mandate for the development of interventions aimed at alleviating symptoms. The current study provides a first exploration of who should be offered a VR intervention. As a guide to oncology HCPs, we have learnt that VR is particularly engaging and effective among adolescents ( $>13$ years) and females and is more suited to children when they are feeling more well in their treatment cycle. HCPs may consider prioritizing VR for these patients, based on preferences demonstrated, or for patients who report or exhibit higher levels of distress as this would indicate greater need for VR intervention and predictably greater relief of symptoms and mood improvement.

\subsection{Study Limitations}

While the VR equipment used in this study delivered fully-immersive VR experiences, subsequent improvements to VR technology in terms of comfort, weight, resolution and integration means newer models may be more appropriate to increase intervention usability and effectiveness (Mahrer and Gold, 2009). It is also possible that the iPad represented something more novel or engaging than was anticipated for the control condition. Future studies should engage multiple comparison groups to improve our understanding of VR effectiveness relative to other technologybased and usual care approaches. Finally, the VR content was limited to 10-minutes due to significant production costs, possibly impacting the strength of effects. Future studies should evaluate the differential effects of time spent immersed in VR (i.e. dosage) on child outcomes to guide optimal treatment delivery.

\subsection{Conclusions}

Immersive VR, delivered using VR HMD, shows promise as a prevention-focused intervention in supporting mood adjustment in hospitalized pediatric oncology patients. Future studies should continue to explore how Immersive VR therapy may be used with pediatric patients to 
offer respite from the stress of hospitalisation and treatment, bolster resilience, and curb the onset of depressive symptoms (e.g. low mood, withdrawal, anhedonia). As VR systems continue to advance, replication with equipment with even greater immersive capacity is expected to produce greater effects on psychological targets. The influence of age, sex and treatment differences should continue to be examined to support recommendations regarding patients' suitability for VR interventions in practice. In addition, the efficacy of VR on secondary treatment benefits, such as compliance and satisfaction with care, should be explored so that they may be used to support investment and adoption of VR technology by health professionals. 


\section{References:}

Carlson, K. L., Broome, M. \& Vessey, J. A. 2000. Using Distraction to Reduce Reported Pain, Fear, and Behavioral Distress in Children and Adolescents: A Multisite Study. Journal for Specialists in Pediatric Nursing, 5, 75-85. doi:10.1111/j.1744-6155.2000.tb00089.x.

Chan, E. A., Chung, J. W. Y., Wong, T. K. S., Lien, A. S. Y. \& Yang, J. Y. 2007. Application of a virtual reality prototype for pain relief of pediatric burn in Taiwan. Journal of clinical nursing, 16, 786-793. doi:10.1111/j.1365-2702.2006.01719.x.

Chirico, A., Lucidi, F., De Laurentiis, M., Milanese, C., Napoli, A. \& Giordano, A. 2016. Virtual Reality in Health System: Beyond Entertainment. A Mini-Review on the Efficacy of VR During Cancer Treatment. Journal of Cellular Physiology, 231, 275-287. doi:10.1002/jcp.25117.

Dascal, J., Reid, M., Ishak, W. W., Spiegel, B., Recacho, J., Rosen, B. \& Danovitch, I. 2017. Virtual Reality and Medical Inpatients: A Systematic Review of Randomized, Controlled Trials. Innovations in Clinical Neuroscience, 14, 14-21. https://www.ncbi.nlm.nih.gov/pmc/articles/PMC5373791/

Eijlers, R., Utens, E. M. W. J., Staals, L. M., De Nijs, P. F. A., Berghmans, J. M., Wijnen, R. M. H., Hillegers, M. H. J., Dierckx, B. \& Legerstee, J. S. 2019. Meta-Analysis: Systematic Review and Meta-analysis of Virtual Reality in Pediatrics: Effects on Pain and Anxiety. Anesthesia and analgesia, 129, 1344-1353. doi:10.1213/ane.0000000000004165.

Georgiou, Y. \& Kyza, E. A. 2017. The development and validation of the ARI questionnaire: An instrument for measuring immersion in location-based augmented reality settings. International Journal of Human-Computer Studies, 98, 24-37. doi:10.1016/j.ijhcs.2016.09.014 
Gershon, J., Zimand, E., Pickering, M., Rothbaum, B. O. \& Hodges, L. 2004. A pilot and feasibility study of virtual reality as a distraction for children with cancer. Journal of the American Academy of Child and Adolescent Psychiatry, 43, 1243-1249. doi:10.1097/01.chi.0000135621.23145.05.

Gift, A. G. 1989. Visual analogue scales: measurement of subjective phenomena. Nursing research, 38, 286-288. doi:10.1097/00006199-198909000-00006.

Gold, J. I., Belmont, K. A. \& Thomas, D. A. 2007. The neurobiology of virtual reality pain attenuation. Cyberpsychology and Behavior, 10, 536-544. doi:10.1089/cpb.2007.9993.

Gold, J. I., Kim, S. H., Kant, A. J., Joseph, M. H. \& Rizzo, A. S. 2006. Effectiveness of Virtual Reality for Pediatric Pain Distraction during IV Placement. CyberPsychology \& Behavior, 9, 207-212. doi:10.1089/cpb.2006.9.207.

Harris, P. A., Taylor, R., Thielke, R., Payne, J., Gonzalez, N. \& Conde, J. G. 2009. Research electronic data capture (REDCap) — a metadata-driven methodology and workflow process for providing translational research informatics support. Journal of biomedical informatics, 42, 377-381. doi:10.1016/j.jbi.2008.08.010.

Hoeft, R. M., Vogel, J. \& Bowers, C. A. 2003. Kids get sick too: a proposed child simulator sickness questionnaire. In Proceedings of the Human Factors and Ergonomics Society Annual Meeting. Sage CA: Los Angeles: CA: SAGE Publications doi:10.1177/154193120304702013

Hooke, M. C. \& Linder, L. A. 2019. Symptoms in Children Receiving Treatment for Cancer-Part I: Fatigue, Sleep Disturbance, and Nausea/Vomiting. Journal of Pediatric Oncology Nursing, 36, 244-261. doi:10.1177/1043454219849576.

Hua, Y., Qiu, R., Yao, W.-Y., Zhang, Q. \& Chen, X.-L. 2015. The Effect of Virtual Reality Distraction on Pain Relief During Dressing Changes in Children with Chronic Wounds on Lower Limbs. Pain Management Nursing, 16, 685-691. doi:10.1016/j.pmn.2015.03.001. 
Jeffs, D., Dorman, D., Brown, S., Files, A., Graves, T., Kirk, E., Meredith-Neve, S., Sanders, J., White, B. \& Swearingen, C. J. 2014. Effect of virtual reality on adolescent pain during burn wound care. Journal of Burn Care \& Research, 35, 395-408. doi:10.1097/BCR.00000000000000019.

Kenney, M. P. \& Milling, L. S. 2016. The effectiveness of virtual reality distraction for reducing pain: A meta-analysis. Psychology of Consciousness: Theory, Research, and Practice, 3, 199210. doi: $10.1037 / \mathrm{cns} 0000084$

10.1037/cns0000084.supp (Supplemental).

Kipping, B., Rodger, S., Miller, K. \& Kimble, R. M. 2012. Virtual reality for acute pain reduction in adolescents undergoing burn wound care: a prospective randomized controlled trial. Burns, 38, 650-657. doi:10.1016/j.burns.2011.11.010.

Krijn, M., Emmelkamp, P. M. G., Olafsson, R. P. \& Biemond, R. 2004. Virtual reality exposure therapy of anxiety disorders: A review. Clinical psychology review, 24, 259-281. doi:10.1016/j.cpr.2004.04.001.

Li, W. H., Chung, J. O. \& Ho, E. K. 2011. The effectiveness of therapeutic play, using virtual reality computer games, in promoting the psychological well-being of children hospitalised with cancer. 20, 2135-2143. doi:10.1111/j.1365-2702.2011.03733.x.

Linder, L. A. \& Hooke, M. C. 2019. Symptoms in Children Receiving Treatment for Cancer-Part II: Pain, Sadness, and Symptom Clusters. Journal of Pediatric Oncology Nursing, 36, 262279. doi:10.1177/1043454219849578.

Mahrer, N. E. \& Gold, J. I. 2009. The use of virtual reality for pain control: A review. Current Pain and Headache Reports, 13, 100-109. doi:10.1007/s11916-009-0019-8.

Mcallister, E., Bhullar, N. \& Schutte, N. S. 2017. Into the woods or a stroll in the park: how virtual contact with nature impacts positive and negative affect. International journal of environmental research and public health, 14, 786. doi:10.3390/ijerph14070786. 
Parsons, T. D., Rizzo, A. A., Rogers, S. \& York, P. 2009. Virtual reality in paediatric rehabilitation: A review. Developmental Neurorehabilitation, 12, 224-238. doi:10.1080/17518420902991719.

Rourke, M. T., Samson, K. K. \& Kazak, A. E. 2015. Psychological Aspects of Long-Term Survivorship. In Schwartz C., Hobbie W., Constine L., Ruccione K. (eds) Survivors of Childhood and Adolescent Cancer. Pediatric Oncology. Springer, Cham, doi:10.1007/978-3319-16435-9_20.

Sander Wint, S., Eshelman, D., Steele, J. \& Guzzetta, C. E. 2002. Effects of distraction using virtual reality glasses during lumbar punctures in adolescents with cancer. Oncology nursing forum, 29, E8-E15. doi:10.1188.02/ONF.E8-E15.

Schneider, S. M. \& Workman, M. L. 1999. Effects of virtual reality on symptom distress in children receiving chemotherapy. Cyberpsychology and Behavior, 2, 125-134. doi:10.1089/cpb.1999.2.125.

Schulz, K. F., Altman, D. G., Moher, D. \& The, C. G. 2010. CONSORT 2010 Statement: updated guidelines for reporting parallel group randomised trials. BMC Medicine, 8, 18. doi:10.1186/1741-7015-8-18.

Sharar, S. R., Carrougher, G. J., Nakamura, D., Hoffman, H. G., Blough, D. K. \& Patterson, D. R. 2007. Factors influencing the efficacy of virtual reality distraction analgesia during postburn physical therapy: preliminary results from 3 ongoing studies. Archives of physical medicine and rehabilitation, 88, S43-S49. doi:10.1016/j.apmr.2007.09.004.

Slater, M. \& Wilbur, S. 1997. A framework for immersive virtual environments (FIVE): Speculations on the role of presence in virtual environments. Presence: Teleoperators \& Virtual Environments, 6, 603-616. doi:10.1162/pres.1997.6.6.603.

Spence, S., Sawyer, M., Sheffield, J., Patton, G., Bond, L., Graetz, B. \& Kay, D. 2014. Does the absence of a supportive family environment influence the outcome of a universal intervention 
for the prevention of depression? International journal of environmental research and public health, 11, 5113-5132. doi:10.3390/ijerph110505113.

Statacorp 2013. Stata Statistical Software: Release 13. College Station, TX: StataCorp LP.

Valmaggia, L. R., Latif, L., Kempton, M. J. \& Rus-Calafell, M. 2016. Virtual reality in the psychological treatment for mental health problems: An systematic review of recent evidence. Psychiatry Research, 236, 189-195. doi:10.1016/j.psychres.2016.01.015.

Van Schoors, M., Caes, L., Verhofstadt, L. L., Goubert, L. \& Alderfer, M. A. 2015. Systematic Review: Family Resilience After Pediatric Cancer Diagnosis: Figure 1. Journal of Pediatric Psychology, 40, 856-868. doi:10.1093/jpepsy/jsv055.

Varni, J. W., Burwinkle, T. M., Katz, E. R., Meeske, K. \& Dickinson, P. 2002. The PedsQLTM in pediatric cancer: reliability and validity of the pediatric quality of life inventory ${ }^{\mathrm{TM}}$ generic core scales, multidimensional fatigue scale, and cancer module. Cancer, 94, 2090-2106. doi:10.1002/cncr.10428

Wolitzky, K., Fivush, R., Zimand, E., Hodges, L. \& Rothbaum, B. O. 2005. Effectiveness of virtual reality distraction during a painful medical procedure in pediatric oncology patients. Psychology and Health, 20, 817-824. doi:10.1080/14768320500143339.

Won, A., Bailey, J., Bailenson, J., Tataru, C., Yoon, I. \& Golianu, B. 2017. Immersive Virtual Reality for Pediatric Pain. Children, 4, 52. doi:10.3390/children4070052. 


\section{Table 1}

Patient sample characteristics $(N=90)$

Demographic and disease characteristics

\section{Child Age [Mean, SD]}

Younger (7-12)

$60(66.67)$

Older (13-19)

$30(33.33)$

Sex $[\mathrm{n}, \%]$

Male

$50(55.6)$

Female

$40(44.4)$

Country of birth [n, \%]

Australia

$84(93.33)$

Other

$6(6.67)$

Disease type [n, \%]

Leukemia

$40(44.44)$

Lymphoma

$13(14.44)$

Brain tumor/CNS

$4(4.44)$

Bone

$16(17.8)$

Soft tissue

$4(4.44)$

Melanoma

$1(1.11)$

Germ cell

$4(4.44)$

Other immunodeficiency and hematological conditions

$8(8.89)$

Disease relapsed [n, \%]

Yes

$10(11.1)$

No

$80(88.9)$

Treatment history [Mean, SD, range]

Total days since diagnosis

$255.66(338.67) \quad 25-1772$

Total days current admission

$18.69(25.80) \quad 1-140$

Total days as an inpatient past 12 months

$53.70(37.76)$

4-199 
Table 2

Patient screening and outcome measures

\begin{tabular}{|c|c|c|c|}
\hline Construct & Measure & Description & $\begin{array}{l}\text { Time point } \\
\text { administered }^{\text {a }}\end{array}$ \\
\hline \multicolumn{4}{|c|}{$\begin{array}{l}\text { Screening, Demographics, } \\
\text { Treatment variables }\end{array}$} \\
\hline Baseline characteristics & $\begin{array}{l}\text { Demographics, disease } \\
\text { and treatment factors }\end{array}$ & $\begin{array}{l}\text { Participant demographic information (age, sex, country of } \\
\text { birth), diagnosis (disease type, relapse status), and treatment } \\
\text { variables (diagnosis date, length of current admission, total } \\
\text { days hospitalized in past } 12 \text { months) was collected using } \\
\text { hospital electronic medical records. }\end{array}$ & Screening \\
\hline \multicolumn{4}{|l|}{ Primary outcomes } \\
\hline $\begin{array}{l}\text { Child state } \\
\text { Positive mood } \\
\text { Anxiety } \\
\text { Anger } \\
\text { Nausea } \\
\text { Pain }\end{array}$ & $\begin{array}{l}\text { Child-report } \\
\text { Visual Analogue Scale } \\
\text { (VAS) }[\mathrm{mm}]\end{array}$ & $\begin{array}{l}\text { Five VASs developed for this study, were used to assess } \\
\text { child emotional and physical states (refer to supplementary } \\
\text { material, Figures S2-S6). } \\
\text { Formatted as } 100 \mathrm{~cm} \text { horizontal lines anchored at each end } \\
\text { by a contrasting face and thought bubble containing a short } \\
\text { phrase, which indicated no symptoms at the lowest anchor } \\
\text { point and (i.e. 0) and the extreme of the condition at the } \\
\text { highest anchor point (i.e. 10). Participants were asked to } \\
\text { "Place a mark on the line to indicate how you feel now". } \\
\text { Participants could tap anywhere on the line and then make } \\
\text { any adjustments by sliding the marker up or down the scale. } \\
\text { The VASs were scored by measuring the distance (in } \\
\text { millimeters) between the lowest anchor point and mark }\end{array}$ & Pre, post \\
\hline
\end{tabular}


made by the patient. This method has been shown to be sensitive to small changes over time (Gift, 1989).

Pulse rate

Pulse oximeter $\left[\mathrm{SpO}_{2}\right]$

Child-report

Spence Children's

Anxiety Scale

(SCAS)- short form

(Spence et al., 2014)

Child illness status
Parent-proxy report Pediatrics Quality of Life 3.0- cancer module

(PedsQL) (Varni et al., 2002)
8 -items assessing symptoms of child anxiety. Participants are asked to select a word that shows how often each item happens to them; e.g. e.g. "I worry about things" rated on a four-point scale $(0=$ Never, $2=$ Sometimes, $3=$ Often, $4=$ Always). Scores are tallied with higher scores reflecting greater symptoms.

27-items assessing health-related quality of life in children and adolescents with cancer across 8 dimensions: Pain and hurt, Nausea, Procedural anxiety, Treatment anxiety, Worry, Cognitive problems, Perceived physical appearance,

Communication. Items (e.g. "Have a lot of pain"; "Feeling too nauseated to eat") are rated on a five-point Likert scale $(0=$ Never a problem, $1=$ Almost never a problem, $2=$ Sometimes a problem, $3=$ Often a problem, $4=$ Almost always a problem). Higher scores indicate poorer quality of life.
Pre, during, post

Pre

Pre 
Immersion

Enjoyment

Simulator sickness
Child-report

Adapted version of the

Total Immersion

subscale of the

Augmented Reality

Immersion (ARI)

questionnaire

(Georgiou and Kyza,

2017)

Child-report

VAS- Enjoyment

thermometer

Child-report

Child Simulation

Sickness

Questionnaire (CSSQ)

(Hoeft et al., 2003) 7-items for adolescents (13 and above), assessed total

Post

immersion level achieved with intervention. Included two

lower-order constructs founded in VR research: Presence

(the 'feeling of being there' in a digital environment) and

Flow (the psychological state of being so involved in an activity that nothing can distract you). Minor modifications

to item wording were made to enhance item comprehension

by younger children (i.e., aged 7 to 12 years). Items (e.g. "I felt that what I was experiencing was something real rather than something imaginary"; "I was not distracted by my thoughts or other things happening around me during the activity") are rated a five-point Likert scale (1= disagree, 2 $=$ disagree a little bit, $3=$ not sure, $4=$ agree a little bit, $5=$ agree). Higher scores indicated greater immersion (i.e.

absorption in the activity) (refer to supplementary material).

VAS assessing enjoyment level with intervention use. Formatted as a vertically presented "enjoyment thermometer", participants were instructed to rate their experience by giving it a score out of $10(0=I t$ didn't interest me at all, $10=$ I really enjoyed it, it was the best). Higher scores indicated greater satisfaction and enjoyment.

7-items assessing symptoms of simulator sickness in the study of virtual environments across three categories: nausea (i.e. motion sickness), oculomotor (i.e. eye strain) and disorientation (i.e. dizziness). Participants are asked to answer item questions according to how you feel right now; e.g. "Do your eyes hurt?"; "Do you have any upset stomach?" rated on a three-point scale $(0=N o, 2=A$ little, 
$3=A$ lot $)$. Scores are tallied under each category (3-items in each), with a score $>3$ or more in any category signaling the child experiences significant symptoms of simulator

induced sickness.

${ }^{\mathrm{a}}$ Screening = performed by researcher; pre = prior to intervention; during = at the midpoint of intervention use (i.e. 5-minutes); post =

immediately after completion of the intervention (i.e. 10 -minutes). ${ }^{b}=$ age, sex, and length of current admission were included in moderation

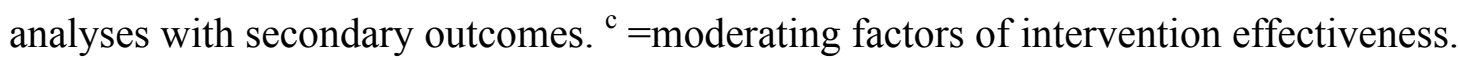




\section{Table 3}

Baseline sample characteristics, child state and randomization grouping between primary intervention groups

\begin{tabular}{lll}
\hline Pre-intervention measures & VR $(\mathbf{n}=\mathbf{6 1})$ & iPad $(\mathbf{n}=\mathbf{2 9})$ \\
\hline Child Age [Mean, SD] & $11.59(3.61)$ & $11.60(2.77)$ \\
Sex [n, \%] & & \\
$\quad$ Males & $37(60.66)$ & $13(44.83)$ \\
$\quad$ Females & $24(39.34)$ & $16(55.17)$ \\
Trait Anxiety [Mean, SD] & $6.10(3.38)$ & $7.24(3.27)$ \\
Length of Admission [n, \%] & & \\
$\quad<=2$ weeks & $37(60.66)$ & $16(55.17)$ \\
$\quad>2$ weeks & $24(39.34)$ & $13(44.83)$ \\
Illness status [Mean, SD] & $60.38(13.94)$ & $58.33(12.70)$ \\
Pre-intervention Child state (VAS mm) [Mean, SD] & & \\
$\quad$ Positive mood & $66.33(22.61)$ & $63.59(24.45)$ \\
$\quad$ Anxiety & $21.21(23.50)$ & $19.55(22.99)$ \\
$\quad$ Anger & $10.28(16.37)$ & $11.72(21.16)$ \\
$\quad$ Nausea & $21.08(24.34)$ & $30.48(30.81)$ \\
$\quad$ Pain & $21.20(25.25)$ & $25.31(24.15)$ \\
Content Assignment [n, \%] & & \\
Nature & & \\
Animals & $22(36.07)$ & $9(31.03)$ \\
Travel & $19(31.15)$ & $10(34.48)$ \\
& $20(32.79)$ & $10(34.48)$ \\
\hline
\end{tabular}


Table 4

Group mean differences in post intervention scores after adjustment* for baseline differences

\begin{tabular}{|c|c|c|c|c|c|}
\hline & VR [Mean, SD] & iPad [Mean, SD] & $\begin{array}{r}\text { Difference } \\
\text { (iPad - } \\
\text { VR) }\end{array}$ & Cohen's $d$ & $P$ value \\
\hline \multicolumn{6}{|l|}{ Pre-post measures } \\
\hline \multicolumn{6}{|c|}{ Child state (VAS mm) } \\
\hline Positive Mood & $82.24(17.91)$ & $75.40(18.07)$ & -6.83 & 0.380 & 0.100 \\
\hline Anxiety & $10.20(15.26)$ & $11.60(15.44)$ & 1.40 & -0.091 & 0.692 \\
\hline Anger & $4.74(8.49)$ & $6.00(8.56)$ & 1.26 & -0.147 & 0.520 \\
\hline Nausea & $14.87(16.57)$ & $17.14(16.77)$ & 2.28 & -0.136 & 0.553 \\
\hline Pain & $10.97(11.23)$ & $12.82(11.34)$ & 1.85 & -0.165 & 0.475 \\
\hline Pulse rate $\left(\mathrm{SpO}_{2}\right)$ & $101.02(7.13)$ & $101.61(7.01)$ & 0.58 & -0.082 & 0.719 \\
\hline \multicolumn{6}{|l|}{ Post measures } \\
\hline Immersion & $29.27(5.46)$ & $28.16(5.51)$ & -1.16 & 0.211 & 0.358 \\
\hline Enjoyment & $8.69(1.85)$ & $8.21(1.87)$ & -0.48 & 0.261 & 0.257 \\
\hline
\end{tabular}

*Note, all analyses adjusted for baseline assessment of outcome, content group, age, gender, trait anxiety, length of admission, functional status 


\section{Figures}
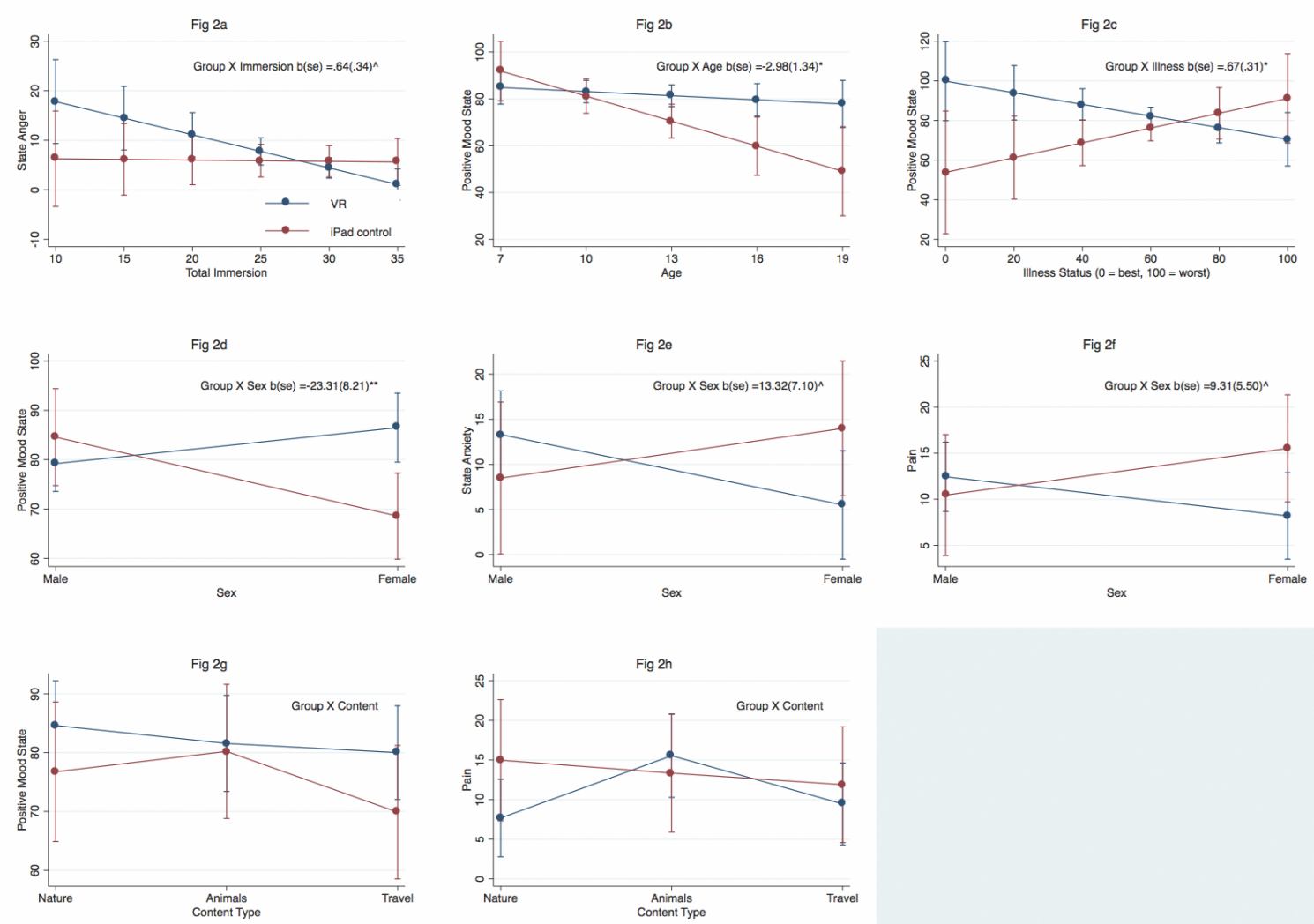

Figure 1. Simple slopes analyses of significant moderation effects found for child post-intervention primary outcomes.

Note: the significance level of the interaction effects presented $={ }^{* * *}<.001,{ }^{* *}<.01,{ }^{*}<.05,{ }^{\wedge}<.1$. See full text for the results of the simple slopes tests. In figures $2 \mathrm{~g}$ and $2 \mathrm{~h}$, interaction effects were non-significant $=p>.05$. 


\section{Supplementary Material}

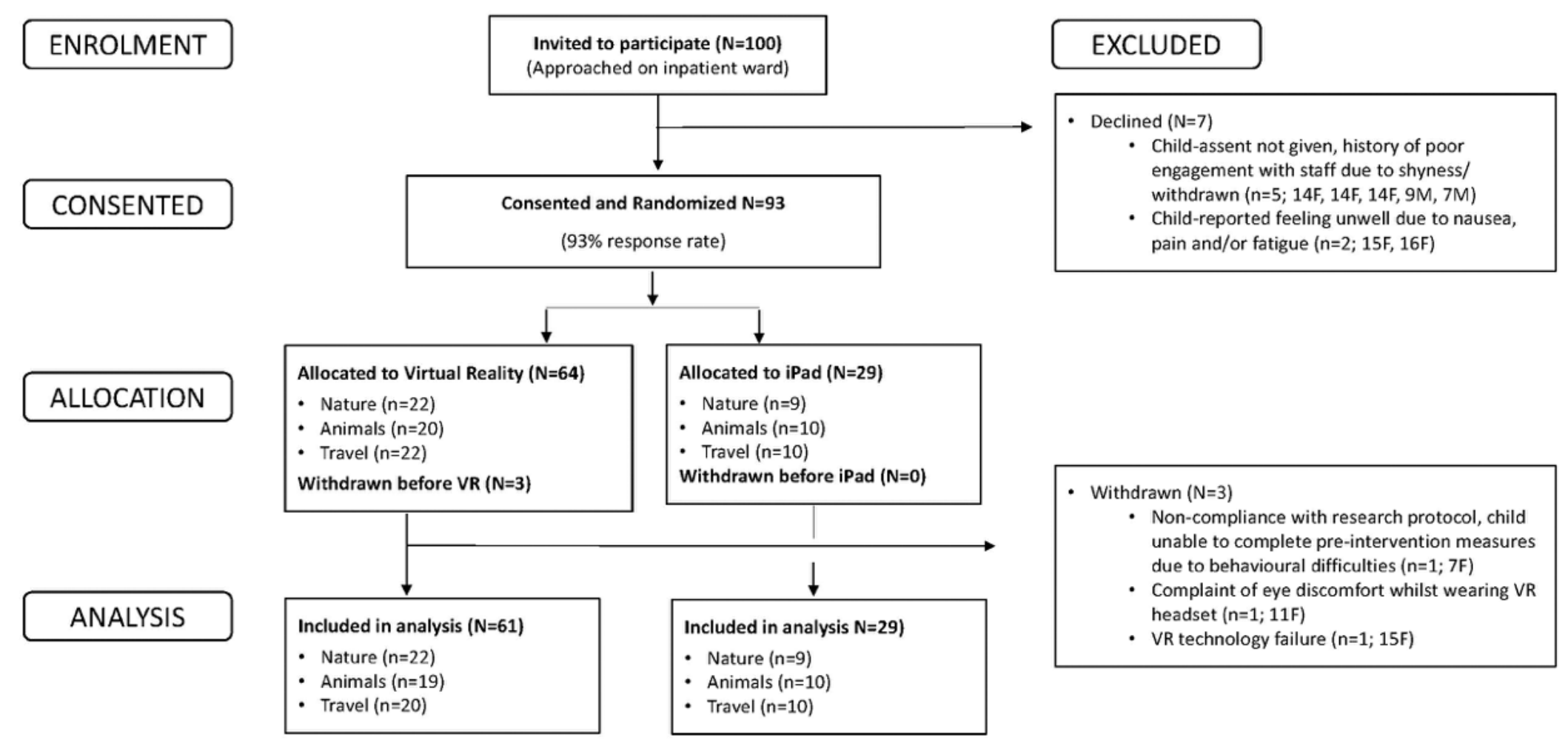

Figure S1. Study CONSORT diagram 
VAS- MOOD

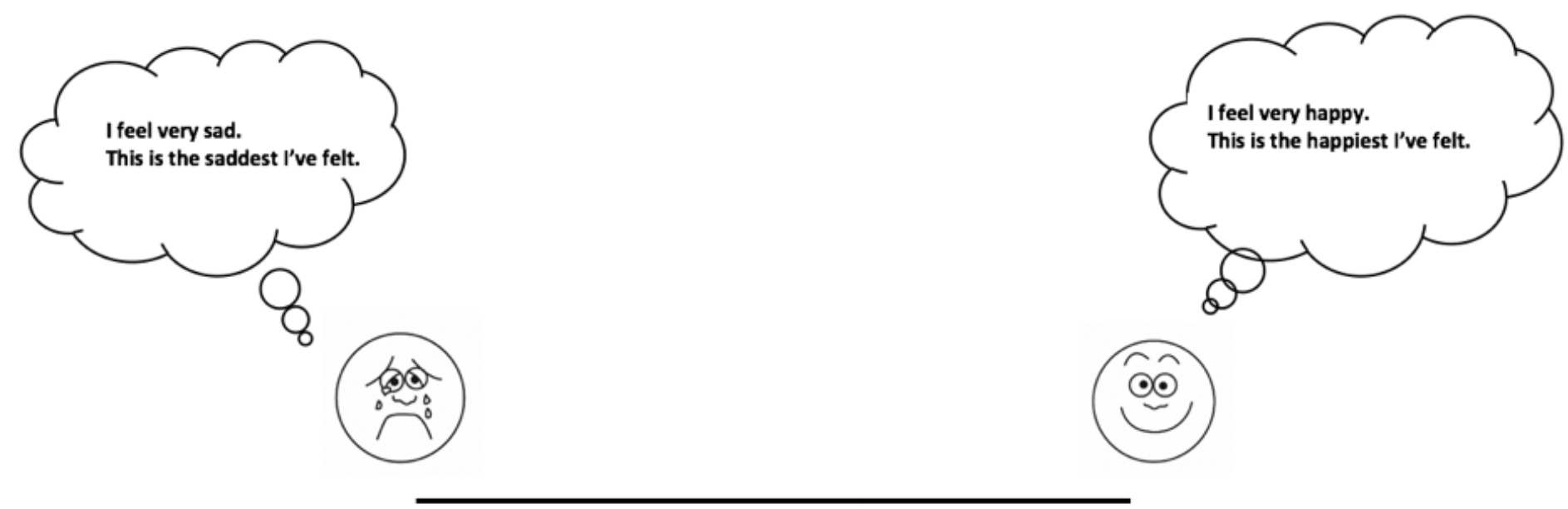

Draw a mark on the line to indicate how you feel now.

Figure S2. Visual Analogue Scale- Positive Mood 


\section{VAS-ANXIETY}
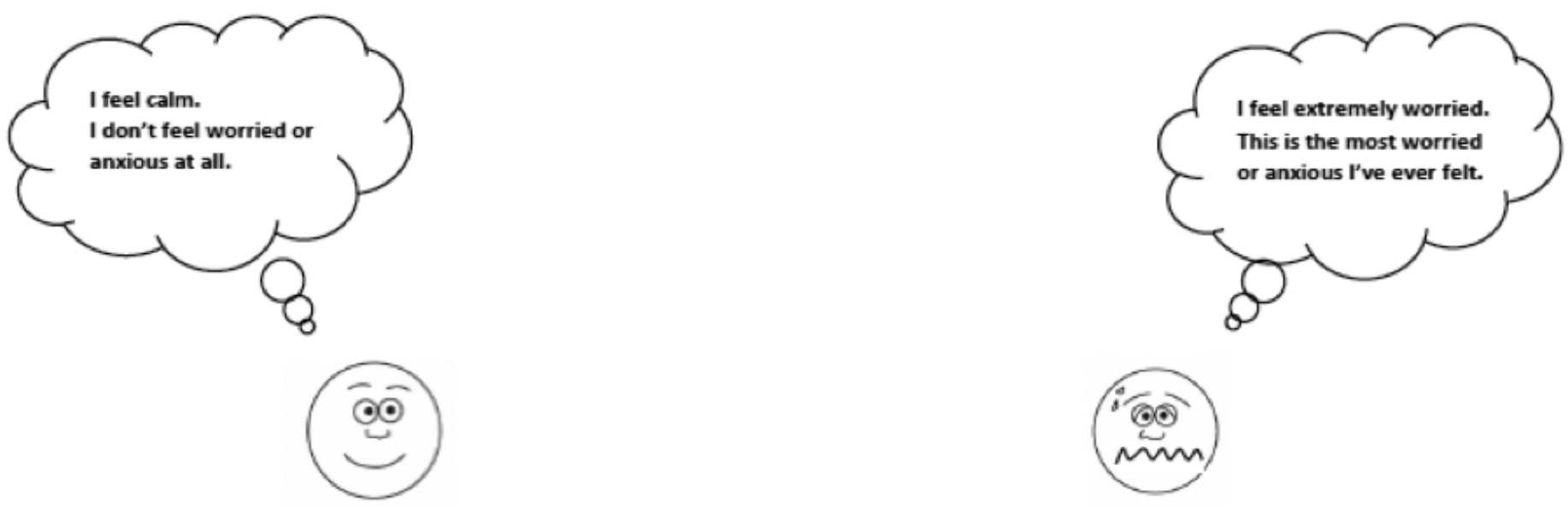

Draw a mark on the line to indicate how worried or anxious you feel now.

Figure S3. Visual Analogue Scale- State Anxiety 
VAS- ANGER
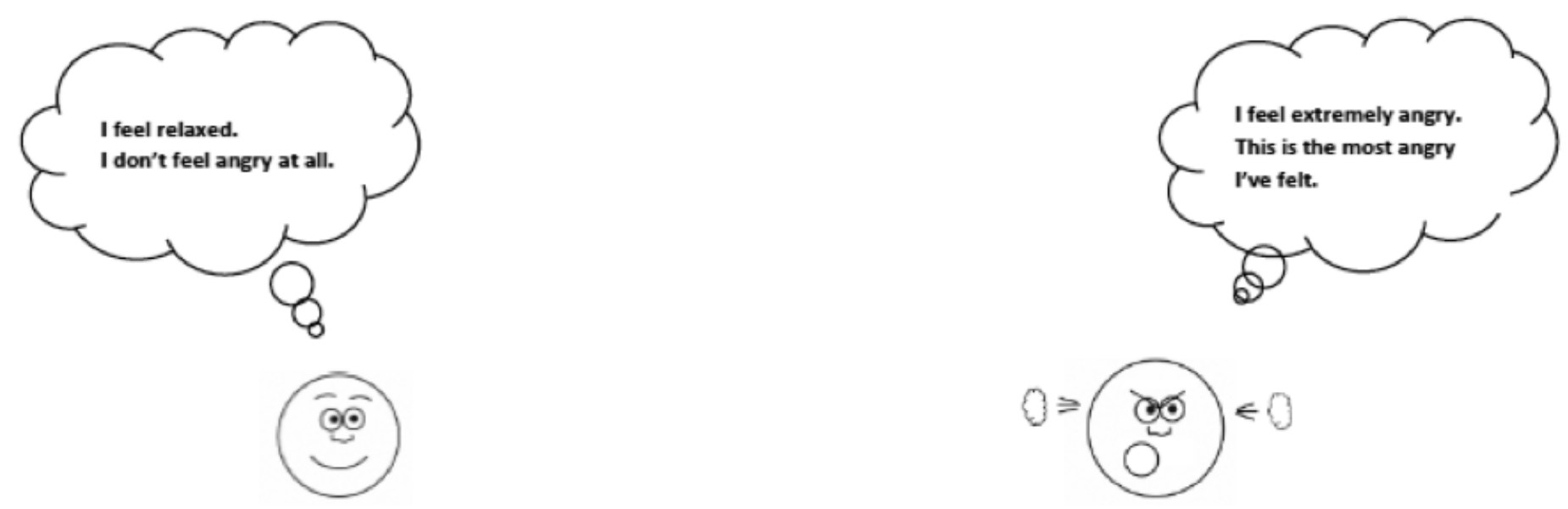

Draw a mark on the line to indicate how much anger you feel now.

Figure S4. Visual Analogue Scale- Anger 


\section{VAS- NAUSEA}
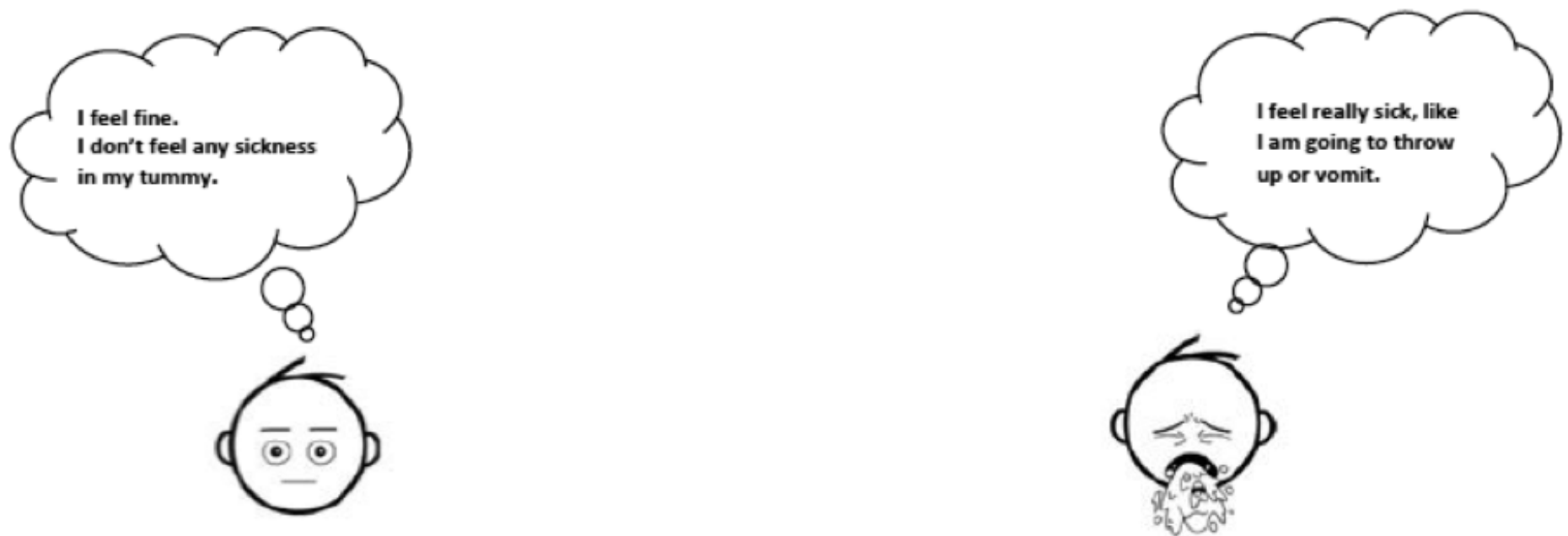

Draw a mark on the line to indicate how you sick or nauseous you feel now.

Figure S5. Visual Analogue Scale- Nausea scale 
VAS- PAIN

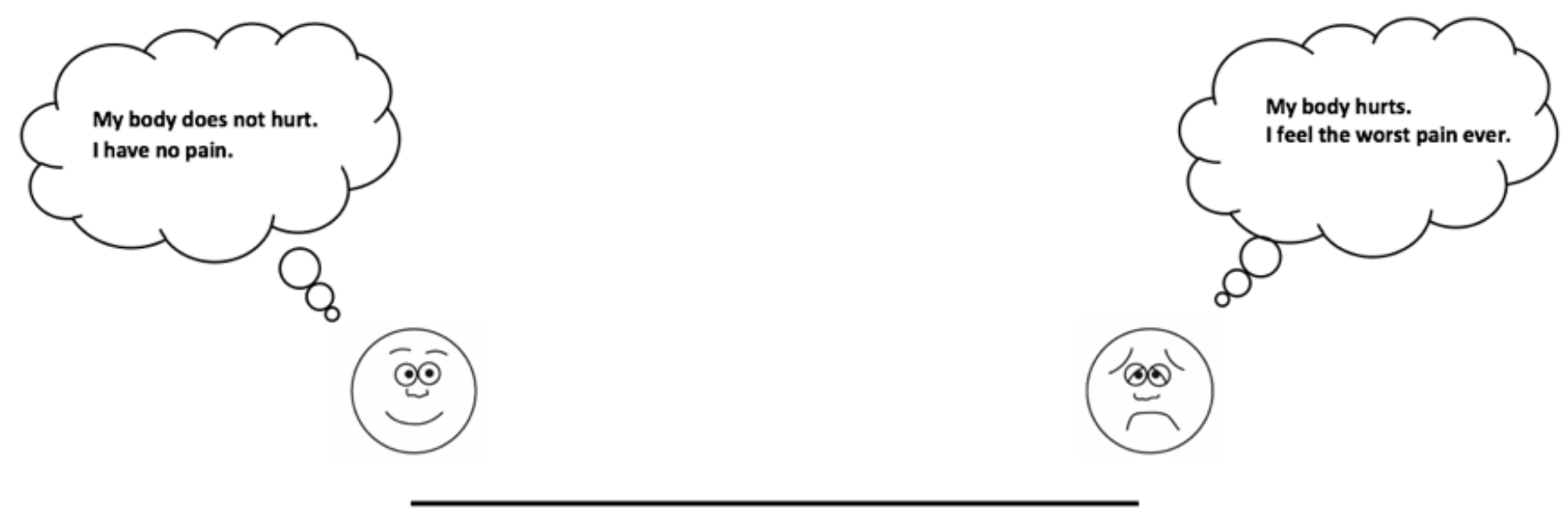

Draw a mark on the line to indicate how much pain you feel now.

Figure S6. Visual Analogue Scale- Pain 
Augmented Reality Immersion (ARI) Questionnaire (Georgiou and Kyza, 2017)

Total immersion - subscale (adapted)

1. The activity felt so similar to real life, that it made me think that the things I saw were real

2. I felt that what I was experiencing was something real rather than something imaginary

3. I was so involved in the activity that sometimes I wanted to reach out and touch things

4. I was so involved that I felt like I could control or change things in the activity

5. I was not distracted by my thoughts or other things happening around me during the activity

6. The activity became the only thing I was thinking about

7. I felt like time just stopped and I could only think about what was happening in the activity. 